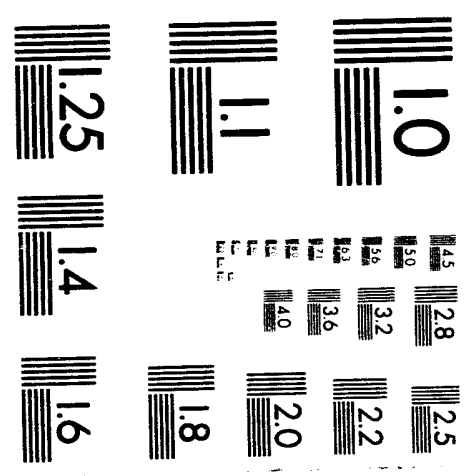



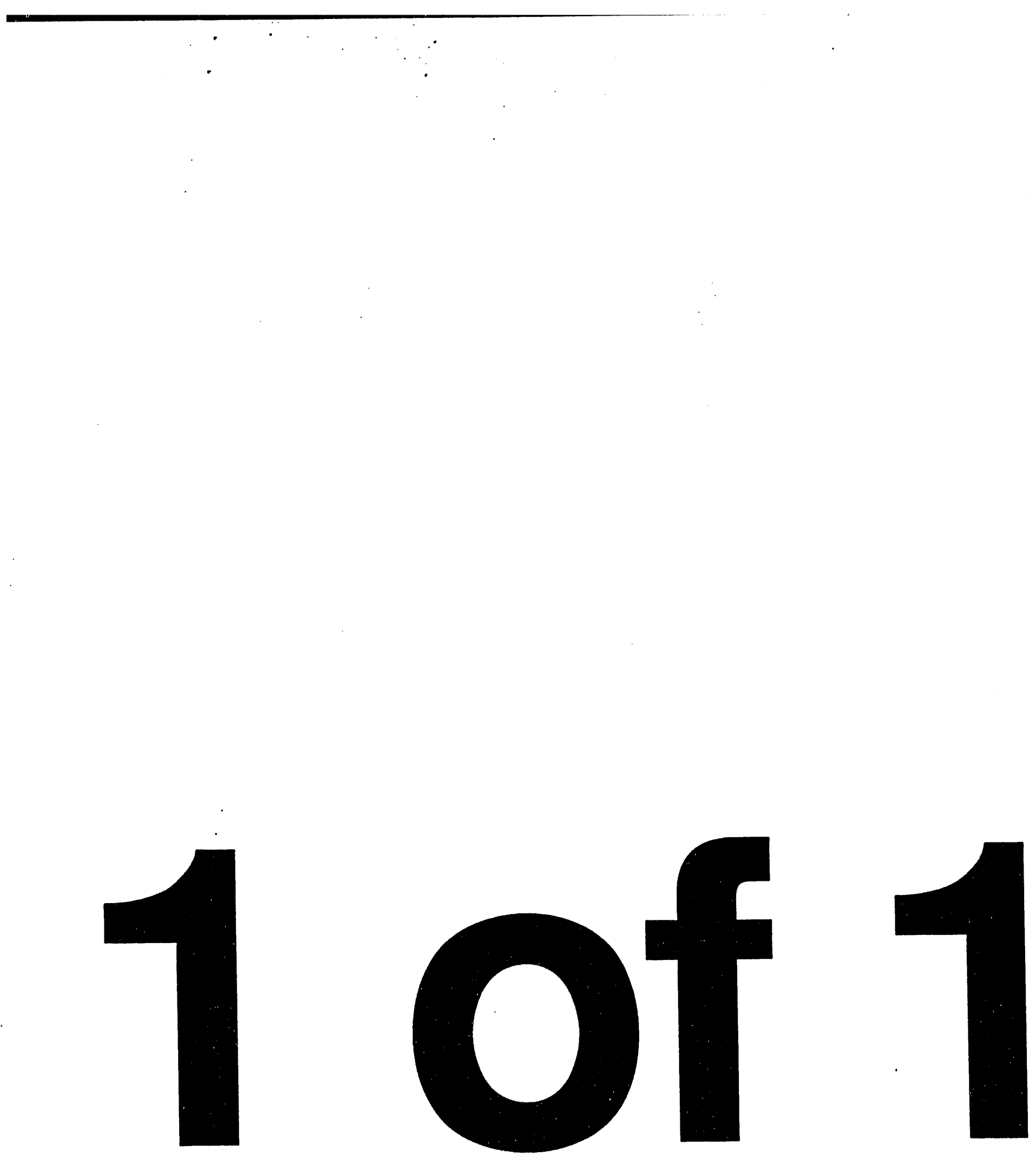
DOE/ER/40561-114-INT93-00-39

\section{Cold Cluster Ferromagnetism}

George F. Bertsch

Institute for Nuclear Theory, $H N-12$, and Department of Physics, FM-15

University of Washington, Seattle, WA 98195

and

Kazuhiro Yabana

Department of Physics, Niigata University, Niigata 950-21, JAPAN

\section{PREPARED FOR THE U.S. DEPARTMENT OF ENERGY UNDER GRANT DE-FG06-90ER40561}

This report was prepared as an account of work sponsored by the United States Government. Neither the United States nor any agency thereof, nor any of their employees, makes any warranty, express or implied, or assumes any legal liability or responsibility for the accuracy, completeness, or usefulness of any information, apparatus, product, or process disclosed, or represents that its use would not infringe privately owned rights. Reference herein to any specific commercial product, process, or service by trade name, mark, manufacturer, or otherwise, does not necessarily constitute or imply its endorsement, recommendation, or favoring by the United States Government or any agency thereof. The views and opinions of authors expressed herein do not necessarily state or reflect those of the United States Government or any agency thereof. 


\title{
Cold Cluster Ferromagnetism
}

\author{
G.F. Bertsch \\ Institute for Nuclear Theory and Dept. of Physics FM-15 \\ University of Washington, Seattle, WA 98195 \\ and K. Yabana \\ Dept. of Physics \\ Niigata University, Niigata 950-21, Japan
}

\begin{abstract}
We examine the magnetic moment distribution of ferromagnetic clusters under conditions where the magnetic moment is aligned with the internal cluster axis. Analytic expressions are obtained for the moment distribution and the adiabatic average moment induced in low fields. The result differs from the low-field Langevin function by a factor $2 / 3$.
\end{abstract}

\section{RECEIVED \\ MAR 031994 \\ OSTI}


Interest has arisen in cluster magnetism with the experimental demonstration of ferromagnetism and superparamagnetism in clusters of several tens or hundreds of atoms[1-5]. In clusters of magnetic elements such as iron, there is a large magnetic moment, which may or may not be frozen into the cluster geometry. At high temperaturs, the moment is free to reorient, and the behavior of the system is call superparamagnetic. In this work we examine the opposite limit, where the moment is aligned along an internal axis. This should be appropriate at low temperatures, and especially for elements that are magnetically "hard", i.e. have high activation energies for reorientation. Parenthetically, we note that the measured behavior of clusters may be more complicated than either of these two limits, with the rotational motion possibly having a dynamic role[5].

We consider a simple model of a spin frozen into a rigid rotor. We also assume that the cluster has equal moments of inertia along all three internal axes. Then the Hamiltonian in an external field $\vec{B}=\hat{z} B$ is given by

$$
H=\frac{\overrightarrow{J^{2}}}{2 I_{0}}+\vec{\mu} \cdot \vec{B}
$$

where $I_{0}$ is the moment of inertia and $\vec{\mu}$ is the magnetic moment vector of the cluster. We denote the magnitude of the moment by $\mu_{0}$. The eigenstates of rotational motion are labeled by the usual $I, K$, and $M$, and the matrix elements of the Hamiltonian in this basis are $<I K M|H| I^{\prime} K^{\prime} M^{\prime}>=\delta_{M, M^{\prime}} \delta_{K, K^{\prime}}\left(\frac{I(I+1)}{2 I_{0}} \delta_{I, I^{\prime}}+\mu_{0} B \sqrt{\frac{(2 I+1)}{\left(2 I^{\prime}+1\right)}}\left(I^{\prime} K^{\prime} 10 \mid I K\right)\left(I^{\prime} M^{\prime} 10 \mid I M\right)\right)$

We shall be concerned here with the distribution of magnetic moments in clusters prepared in molecular beams and measured by deflection in an inhomogeneous magnetic field. We assume that the clusters are produced in a statistical ensemble characterized by a rotational temperature $T$. The magnetic moments change as the cluster enters the magnetic field, but this process is adiabatic. We thus use a canonical ensemble of spins weighted by the zero-field Boltzmann factors. The magnetic moment distribution in a field $B$ is then given by

$$
P(\mu, B)=\frac{\sum_{i} \delta\left(\mu-\mu_{i}(B)\right) \exp \left(-J_{i}\left(J_{i}+1\right) / 2 I_{0} T\right)}{\sum_{J}(2 J+1)^{2} \exp \left(-J(J+1) / 2 I_{0} T\right)}
$$


with

$$
\mu_{i}(B)=d E_{i} / d B
$$

Here the eigenstates are labeled by $i$, their energies by $E_{i}$, and the $z$-component of the magnetic moment by $\mu_{i}$. The $J_{i}$ is the angular momentum of the state specified by adiabatically removing the magnetic field in state $i$. It may be noted that the usual canonical ensemble with Boltzmann factors $\exp \left(-E_{i} / t\right)$ leads in the classical limit to the Langevin formula for the induced moment[6].

Under typical conditions, the angular momentum is large compared to one. For example, for an iron cluster with 50 atoms at a temperature of $15 \mathrm{~K}$, the rms angular momentum is about $\left\langle J^{2}>^{1 / 2} \approx 200\right.$. Thus one can safely evaluate eq. (3) treating $J$ as a continuous variable and replacing the sum by an integral. That is

$$
\sum_{i} \approx \int_{0}^{\infty} d J \int_{-J}^{J} d K \int_{-J}^{J} d M .
$$

This is straightforward to carry out in the limit of zero field, where the magnetic moment is given by

$$
\mu_{i}=\mu_{0}(J K 10 \mid J K)(J M 10 \mid J M)=\mu_{0} \frac{M K}{J(J+1)}
$$

The result is

$$
P(\mu, 0) \approx \frac{1}{2 \mu_{0}} \ln \left(\mu_{0} /|\mu|\right)
$$

This has a singularity at $\mu=0$ which is an artifact of the continuum approximation. The probability distribution is shown in Fig. 1, both in the continuum limit and for an actual sum displayed as a histogram.

We next consider the effect of a weak field on the system, and evaluate the expectation of the moment to first order in the magnetic field strength. We may still label the states by $\mathrm{J}, \mathrm{K}$, and $\mathrm{M}$, using perturbation theory to include the effect of the magnetic field. The first-order contribution to the moment is given by

$$
<i\left|\mu_{z}\right| i>_{(1)}=2 B \sum_{J \prime} \frac{<J_{i} K_{i} M_{i}\left|\mu_{z}\right| J_{i}^{\prime} K_{i} M_{i}>^{2}}{E_{J}-E_{J \prime}}
$$


where the sum has two terms, $J^{\prime}=J \pm 1$. The needed Clebsch-Gordan coefficients are given by

$$
\begin{gathered}
(J M 10 \mid J-1 M)=-\frac{\sqrt{J^{2}-M^{2}}}{J(2 J+1)} \\
(J M 10 \mid J+1 M)=\frac{\sqrt{(J+1)^{2}-M^{2}}}{J(2 J-1)}
\end{gathered}
$$

The average of the moment of states with given value of $J$ is then found to be

$$
\overline{\langle J|\mu| J\rangle}=\frac{B I_{0} \mu_{0}^{2}}{36 J^{2}}
$$

Putting this in the adiabatic statistical ensemble, eq. (2), and replacing the sum over $J$ by an integral, we obtain for the thermal expectation of the moment the expression

$$
<\mu>=\frac{2}{9} \frac{B \mu_{0}^{2}}{T}
$$

This may be compared with the Langevin formula for the expectation of a classical spin in a thermal bath,

$$
<\mu>_{L}=\mu_{0}\left(\operatorname{coth}\left(\frac{\mu_{0} B}{T}\right)-\frac{T}{\mu_{0} B}\right) .
$$

The low-temperature limit of this formula is $\left\langle\mu>_{L} \approx B \mu_{0}^{2} / 3 T\right.$, which differs from eq. (7) by a factor of $3 / 2$. In fact we can obtain the Langevin result if we modify the Boltzmann factor in eq. (2), replacing the rotational energy by the full energy of the state in the magnetic field. Of course this is unphysical for moments induced in isolated clusters because there is no way for the cluster to re-equilibrate with a thermal bath in the magnetic field region.

For higher magnetic fields, we have not been able to find an analytic expression for the moment distribution or its expectation value. We thus turn to numerical solution of the Hamiltonian to see the behavior of the system. We truncate the matrix at some angular momentum $J_{\max }$, and diagonalize it to find the eigenstates and their magnetic moments. It is not necessary to go as high in angular momentum as is found in the physical systems, because the quantization of $J$ does not place an important role when $T I_{0}>>1$. We present results in Figs. 2-4 with $T I_{0}=100$, truncating the Hamiltonian matrix at $J_{\max }=40$. Fig. 2 shows the probability distribution for a moderate field, $\mu_{0} B / T=1$ and Fig. 3 shows the 
results in a high field, $\mu_{0} B / T=4$. The dependence of $\langle\mu\rangle$ on the field strength parameter is shown in Fig. 4.

From these results, we see that the Langevin formula has a qualitative validity for this Hamiltonian, although it is not quantitatively correct. The very complicated behavior of the moment deduced by de Heer thus requires a more complex Hamiltonian. Undoubtedly it is necessary to treat the spin as an independent dynamic variable, having a coupling to the cluster axis that is neither very strong nor very weak. A purely classical treatment has been made in ref. [7]; it would be interesting to see what minimum assumptions about the quantum mechanical Hamiltonian are necessary to describe the behavior.

The sharp peak at $\mu=0$ in the low-field distribution persists to moderate fields, $\mu_{0} B / T \leq$ 1. Most experiments to date have not had the resolution to see this feature. In ref. [4] a structure is seen in the distribution of $\mathrm{Gd}$ clusters that has this behavior. However, the peak is not seen in all clusters, whereas theory requires that it be a universal feature of the distribution in the low-field limit.

We thank G. Hinger for help in the early stages of this work. The research was supported by the Dept. of Energy under Grant FG06-90ER40561. 


\section{REFERENCES}

[1] D.M. Cox, et al., Phys. Rev. B32 (1985) 7290.

[2] W. deHeer, P. Milani, and A. Chatelain, Phys. Rev. Lett. 65 (1990) 488.

[3] J.P. Bucher, D.C. Douglass, and L.A. Bloomfield, Phys. Rev. Lett. 66 (1991) 742.

[4] D.C. Douglass, J.P. Bucher, and L.A. Bloomfield, Phys. Rev. Lett. 68 (1992) 1774; D.C. Douglass, et al., Phys. Rev. B47 (1993) 12874.

[5] I. Billas, J. Becker, and W. de Heer, Z. Phys. D26 (1993) 325.

[6] J.H. Van Vleck, Theory of Electric and Magnetic Sucseptibilities, Oxford (1932), Chap. 2.

[7] P.J. Jensen and K.H. Bennemann, Z. Phys. D26 (1993) 246.

\section{Figure Captions}

Fig. 1 Distribution of magnetic moments for a rigid rotor coupled to an intrinsic spin. The dot-dash line is the limiting formula for zero magnetic field, eq. (5). The histogram shows numerical results for the canonical ensemble of states with $J_{\max } \leq 40$, and a temperature satisfying $T=100 / I_{0}$.

Fig. 2 The numerical results for the distribution of magnetic moments for the ensemble of eq. (3), with the same parameters as in Fig. 1 except for the magnetic field, which has the value $B=T=100 / I_{0}$.

Fig. 3 The distribution of magnetic moments in a high field. Parameters are the same as in Fig. (1) except for the magnetic moment, which is given by $B=4 T=400 / I_{0}$.

Fig. 4 The average magnetic moment as a function of field strength. The solid line shows the Langevin formula, eq. (8), and the dots show the numerical results with $J_{\max }=40$ and $T=100 / I_{0}$. 
Moment distribution $B=0, J_{\max }=40, T=100$

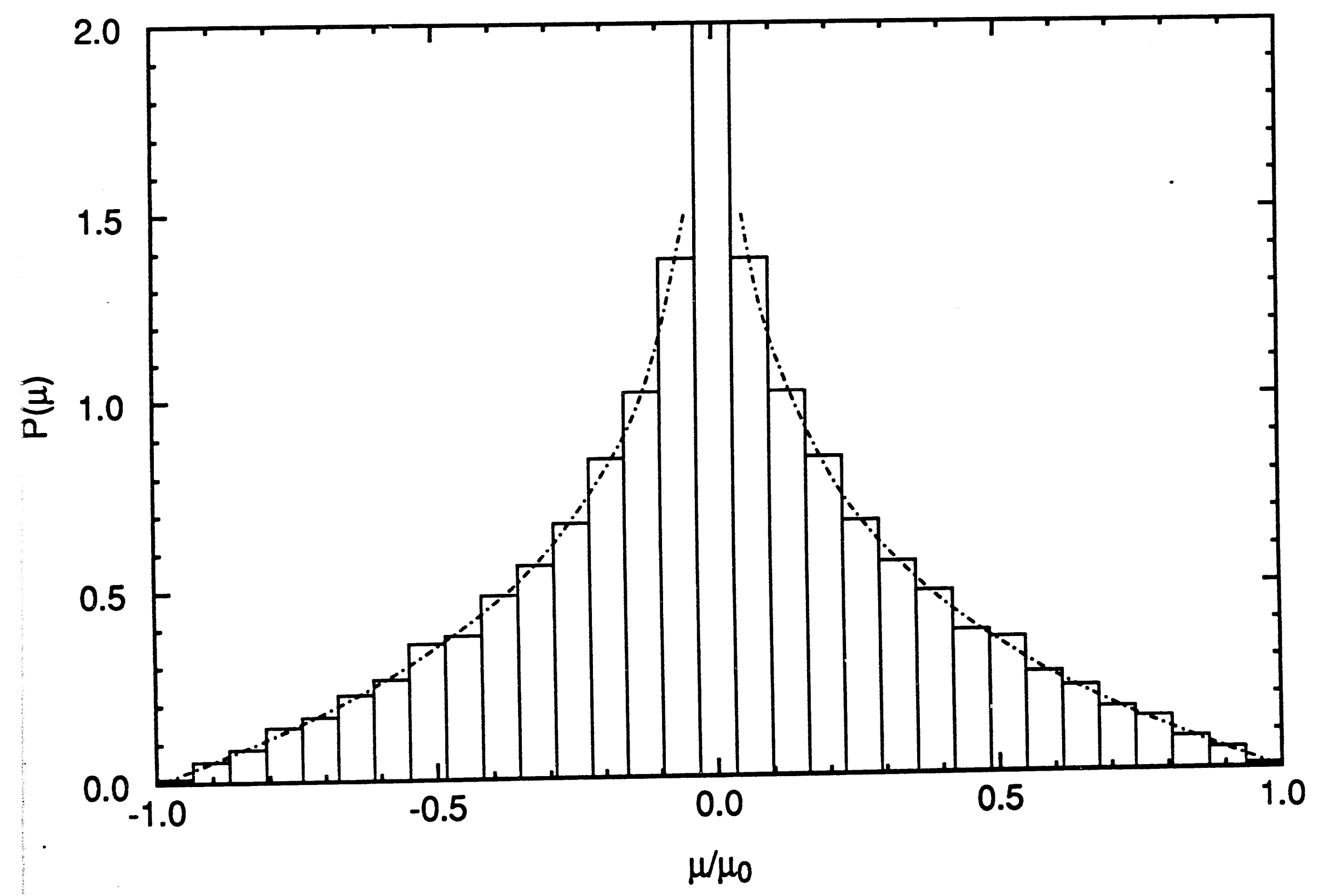




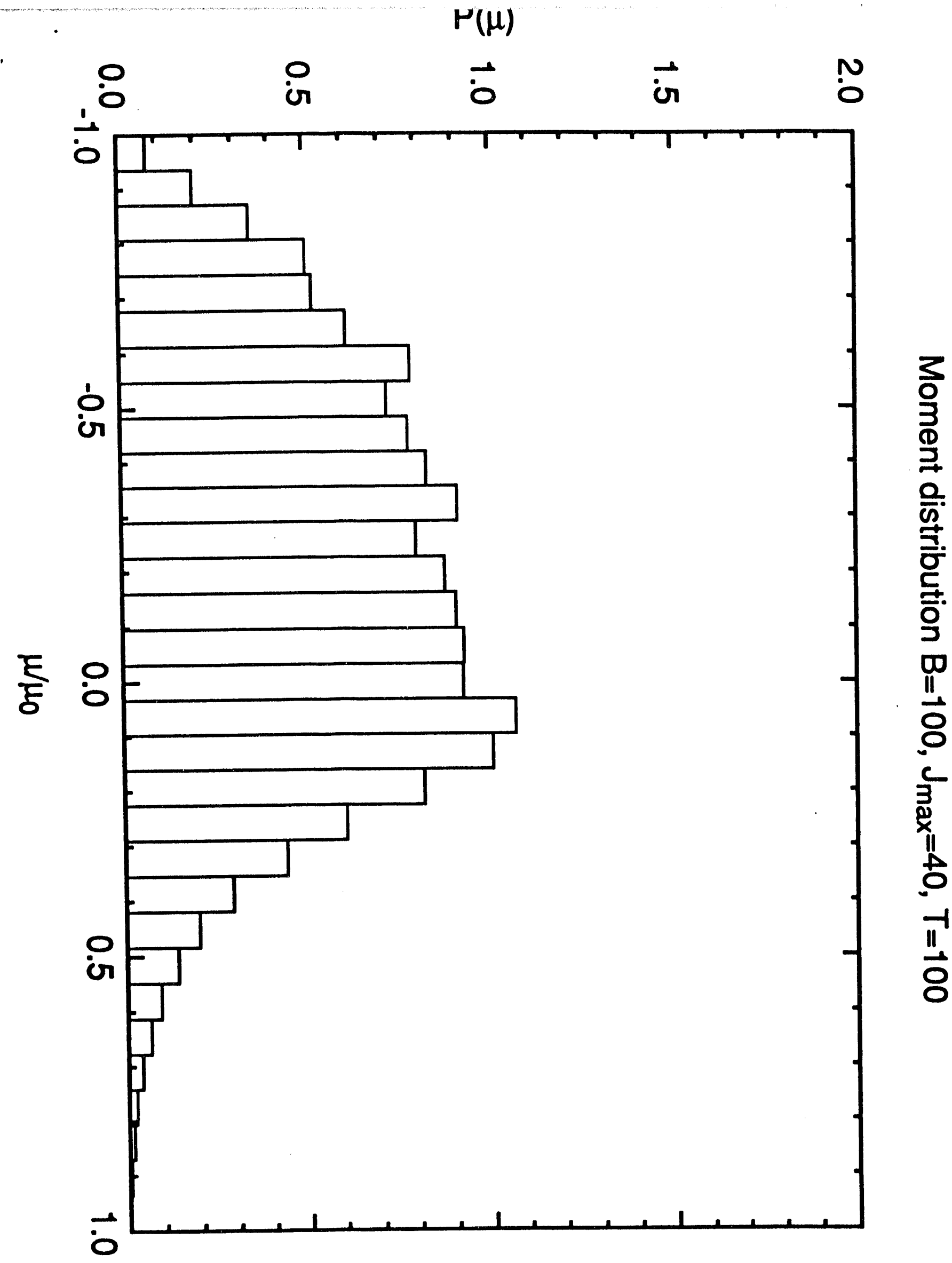

Wed Sep 29, 1900 9:00an Verr:Bertech

Fig. 2 


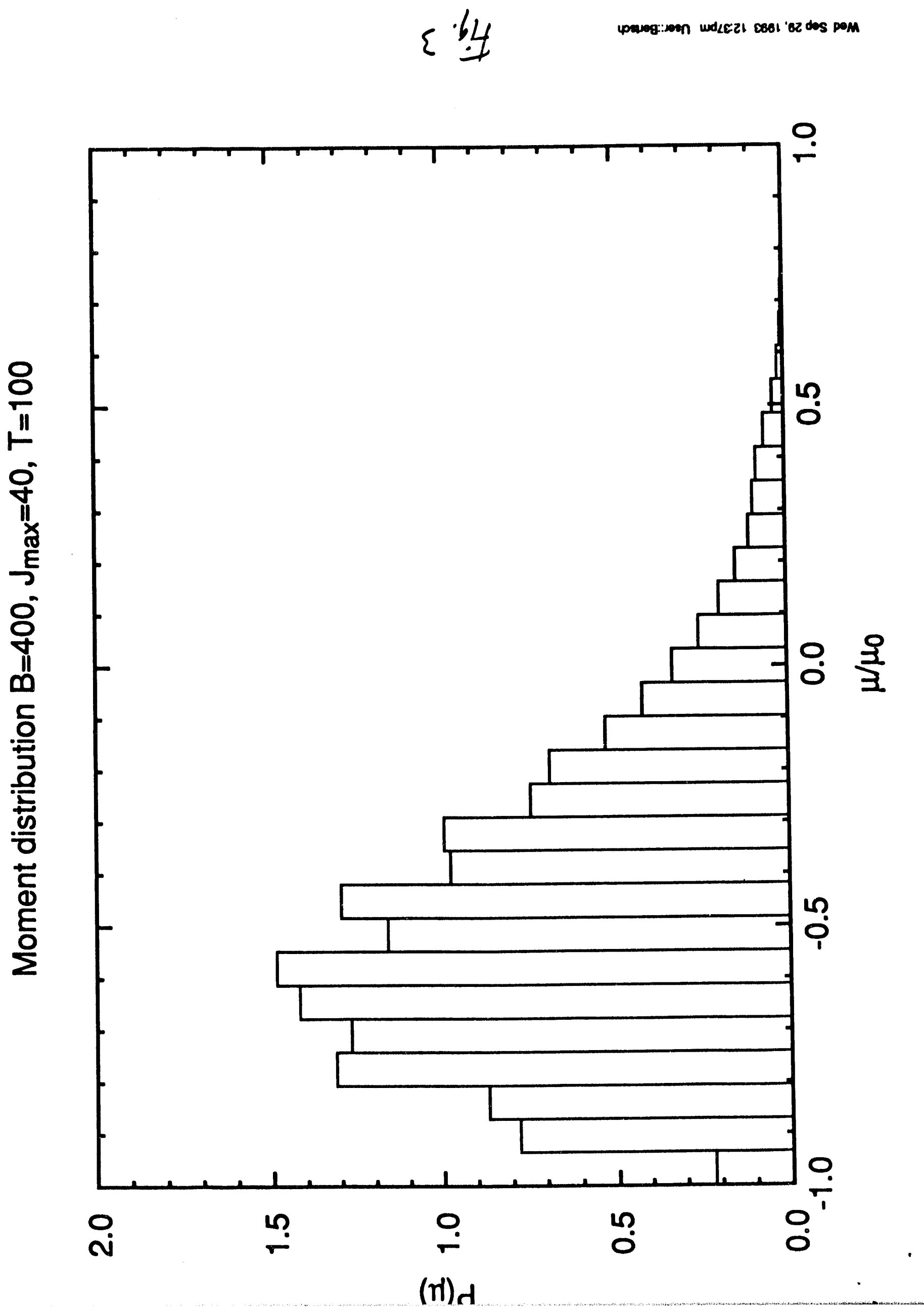



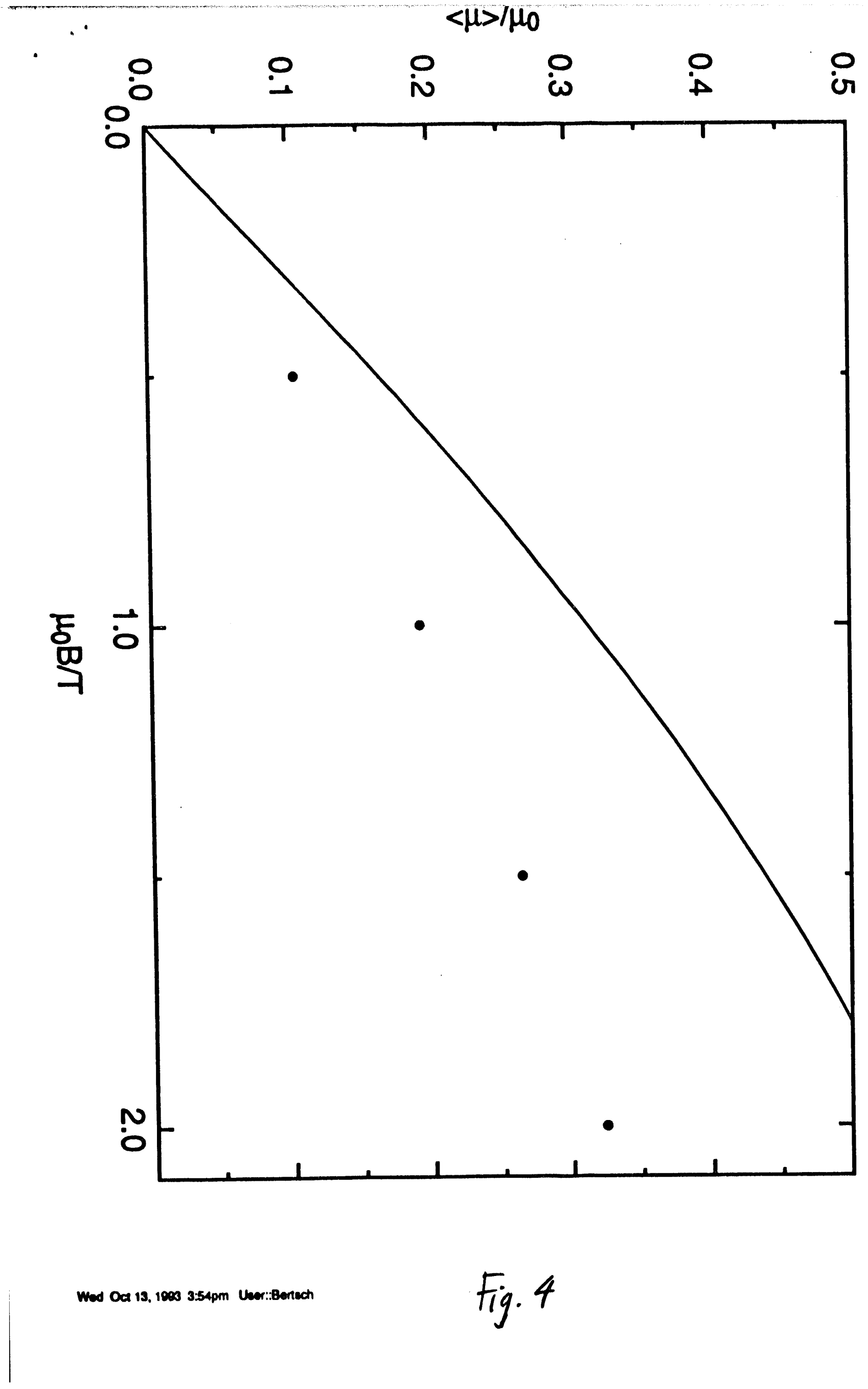

Fig. 4 

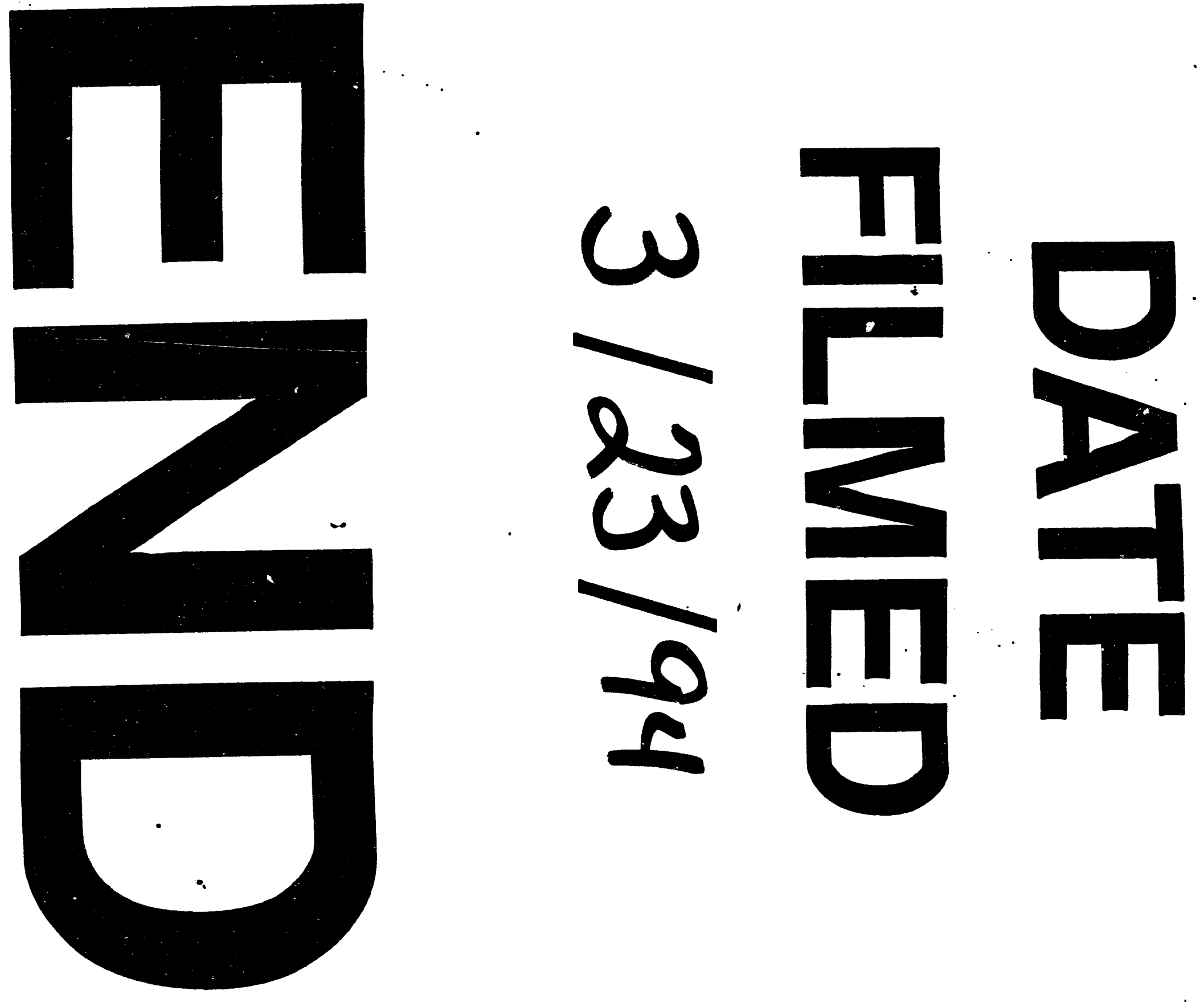
